Supporting Information

For

\title{
Programmed Macromolecular Assembly by Dipole-Dipole Interactions with Aggregation-Induced Enhanced Emission in Aqueous Medium
}

Aritra Rajak and Anindita Das*

*Corresponding author; Email: psuad2@iacs.res.in

School of Applied and Interdisciplinary Sciences, Indian Association for the Cultivation of Science, 2A \& 2B Raja S. C. Mullick Road, Jadavpur, Kolkata-700032, India

Page No

Materials and Methods

Synthesis and Characterization

Experimental Procedures

Additional Figures

NMR Spectra

References 
Materials and Methods: All Chemicals were purchased from commercial suppliers and no further purification was done unless otherwise mentioned. Dried solvents for polymerization were purchased from Sigma-Aldrich. ${ }^{1} \mathrm{H},{ }^{13} \mathrm{C}$ and ${ }^{19} \mathrm{~F}$ NMR spectra were measured in a Bruker $500 \mathrm{MHz}$ and $400 \mathrm{MHz}$ NMR spectrometer using $\mathrm{CDCl}_{3}$ and $\mathrm{D}_{2} \mathrm{O}$ as solvents. Variabletemperature ${ }^{1} \mathrm{H}$ NMR in $\mathrm{D}_{2} \mathrm{O}$ was measured in $300 \mathrm{MHz}$ Bruker NMR spectrometer. Chemical shifts $(\delta)$ are reported in ppm unit with TMS as the internal standard. The coupling constant $(J)$ is reported in hertz $(\mathrm{Hz})$. HRMS were done on XEVO G2-XS Q Tof and MicromassQ-Tof Micro machine. Column chromatography was carried out on silica gel (100-200 mesh). Spectroscopic grade solvents were used for UV-Vis studies, and UV-Vis spectra were recorded in a JASCO V-750 spectrophotometer. Fluorescence spectra were recorded in a FluoroMax-3 spectrophotometer, from Horiba Jobin Yvon. PL lifetime decays were measured using FLS920 steady-state fluorescence spectrometers. Transmission Electron Microscopy (TEM) was performed in JEOL-2010EX machine operating at an accelerating voltage of 200KV. CryoTEM was performed in JEOL JEM-2100 PLUS machine. AFM imaging was performed in an Innova instrument from Bruker \& Asylum Research MFP-3D. FTIR spectra were recorded in a Perkin Elmer Spectrum 100 FT-IR Spectrometer. Olympus IX73 model was used for fluorescence microscopy images. Number average molecular weight $\left(\mathrm{M}_{\mathrm{n}}\right)$ and dispersity (Đ) of the polymers were measured by size exclusion chromatography (SEC) at $30^{\circ} \mathrm{C}$ using a Waters machine equipped with a 515 HPLC pump, Waters 2414 RI detector, and HSP gel HT 4.0/HSP gel HT 2.5 columns connected in a series. THF was used as an eluent. Dynamic Light Scattering (DLS) measurements were recorded in Malvern instrument. 
Scheme 1: Synthesis of $\mathbf{P 1}$ and $\mathbf{P 2}$
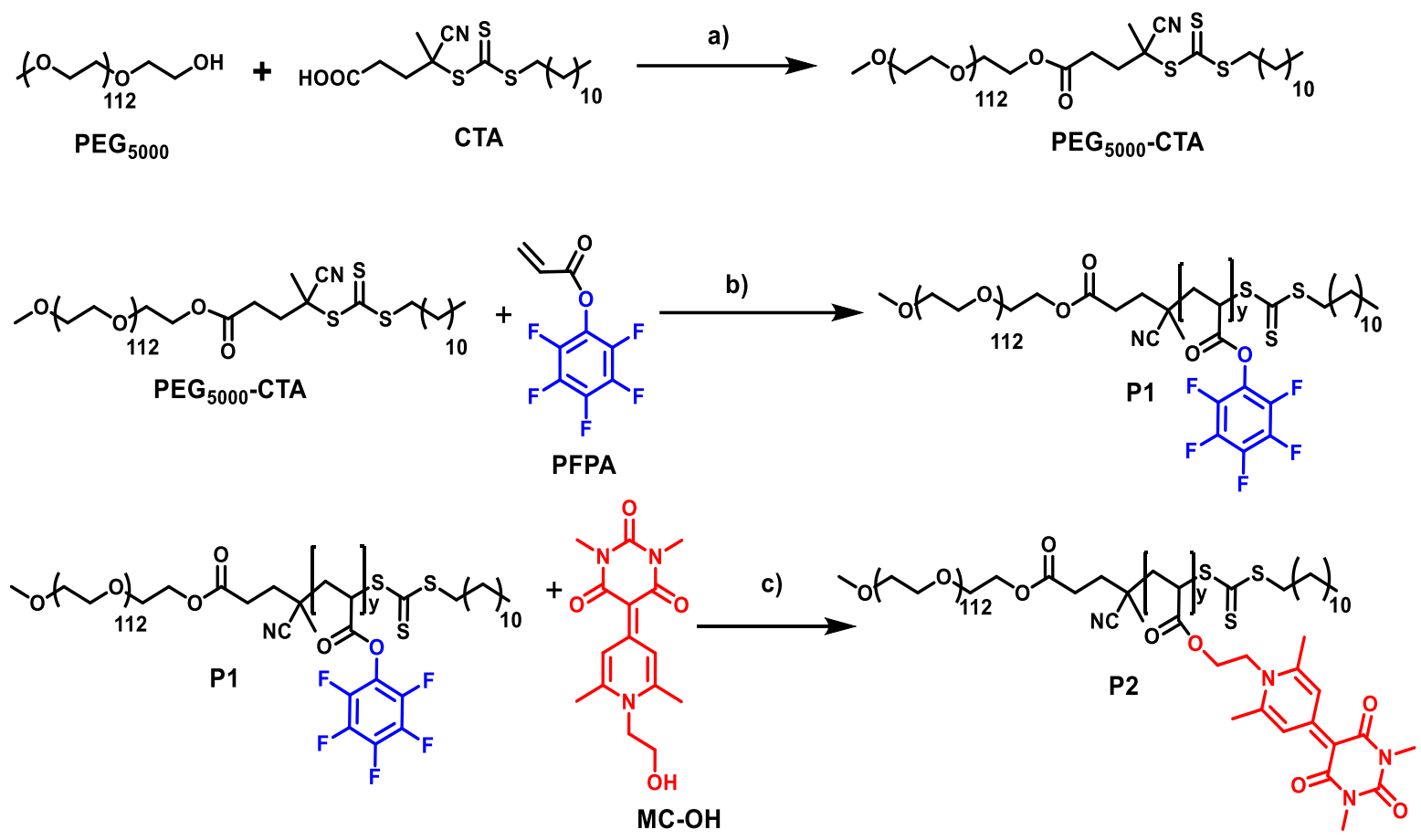

Reagents and conditions: a) dry DCM, DMAP, EDC.HCL, $0{ }^{\circ} \mathrm{C}-\mathrm{rt}, 48 \mathrm{~h}$, yield $=45 \%$; b) AIBN, benzene, $80{ }^{\circ} \mathrm{C}, 24 \mathrm{~h}$, yield $=33 \%$; c) DMF, DMAP, $120^{\circ} \mathrm{C}, 96 \mathrm{~h}$, yield $=85 \%$.

\section{Synthesis and Characterization}

Synthesis of PEGs000-CTA and MC-OH: Synthesized by following our previously reported procedure. ${ }^{1}$

Synthesis of P1: PEG5000-CTA (100 mg, $0.017 \mathrm{mmol}$ ), and pentafluorophenyl acrylate (PFPA) (202 mg, $0.85 \mathrm{mmol})$ were taken along with dry benzene $(1 \mathrm{~mL})$ in a polymer glass vessel equipped with a septum and an inlet/outlet gas tube. The above solution was degassed for $20 \mathrm{~min}$. To this, a solution of azobisisobutyronitrile (AIBN) (0.65 mg, $0.004 \mathrm{mmol})$ in degassed benzene was added and the solution was further degassed for 20 min, Finally the reaction vessel was immersed in a preheated oil bath at $80{ }^{\circ} \mathrm{C}$ for $24 \mathrm{~h}$. The reaction was quenched by exposing to air. The solution was diluted with $\mathrm{CH}_{2} \mathrm{Cl}_{2}$ and purified by precipitating from cold diethyl ether to afford an off- white solid. The process was repeated thrice to obtain a pure polymer which was dried under vacuum at $40{ }^{\circ} \mathrm{C}$ for $24 \mathrm{~h}$ (yield $=100$ $\mathrm{mg}, 33 \%) .{ }^{1} \mathrm{H}$ NMR of $\mathbf{P 1}\left(400 \mathrm{MHz}, \mathrm{CDCl}_{3}\right.$, TMS): $\delta(\mathrm{ppm})=3.82-3.55$ (m, 113H), 3.38 (s, $3 \mathrm{H}), 3.08$ (bs, 1H), 2.47-2.03 (bm, 2H). From the ${ }^{1} \mathrm{H}$ NMR, the degree of polymerization (DP) for PFPA monomer was estimated to be 19. Molar mass calculated from ${ }^{1} \mathrm{H}$ NMR $=9500 \mathrm{~g} /$ mol. FTIR shows characteristic peak for PFP-ester carbonyl stretching at $1783 \mathrm{~cm}^{-1}$. Size exclusion chromatography (SEC) (PS standard, THF): a single monomodal peak appearing at 
a lower retention time with respect to PEG5000-CTA. The dispersity calculated for $\mathbf{P 1}$ from SEC was $Đ=1.48$.

Synthesis of P2: The postpolymer modification of P1 with MC-OH was performed following literature procedure as follows: ${ }^{1,2}$

P1 (70 mg, $0.3 \mathrm{mmol}$ of PFP-ester, 1.0 equiv) and DMAP (7 mg, $0.06 \mathrm{mM}, 0.2$ equiv) were taken with $2.0 \mathrm{~mL}$ dry DMF and added with MC-OH (92 mg, $0.3 \mathrm{mM}, 1.0$ equiv). The mixture was stirred at $120{ }^{\circ} \mathrm{C}$ for $96 \mathrm{~h}$. The formation of the product was monitored by FTIR measurements. The mixture was dissolved in $\mathrm{CH}_{2} \mathrm{Cl}_{2}$ and purified by precipitation from cold hexane. The precipitation method was repeated thrice to obtain solid brown polymer P2 (yield $=60 \mathrm{mg}, 85 \%$ ). The conversion was quantitative as complete disappearance of the PFP-ester carbonyl peak at $1783 \mathrm{~cm}^{-1}$ in the FTIR spectrum and fluorine peaks in ${ }^{19} \mathrm{~F}$ NMR spectrum of P2 was observed after purification of the polymer. ${ }^{1} \mathrm{H}$ NMR $\left(500 \mathrm{MHz}, \mathrm{CDCl}_{3}, \mathrm{TMS}\right): \delta$ (ppm) $=\delta 8.97(\mathrm{~s}, 1 \mathrm{H}), 4.33(\mathrm{bs}, 2 \mathrm{H}), 4.01(\mathrm{bs}, 2 \mathrm{H}), 3.84-3.55(\mathrm{~s}, 113 \mathrm{H}), 3.34(\mathrm{~s}, 6 \mathrm{H}), 2.96(\mathrm{bs}, 1 \mathrm{H})$,

2.64 (s, 6H). From ${ }^{1} \mathrm{H}$ NMR, the degree of polymerization (DP) was estimated to be 11 . The molar mass from the estimated DP was calculated to be $8950 \mathrm{~g} / \mathrm{mol}$.

\section{Experimental procedures:}

Sample preparation in water: Measured quantity of $\mathbf{P 2}$ was transferred from a concentrated stock solution in $\mathrm{CHCl}_{3}$ in a glass vial. The $\mathrm{CHCl}_{3}$ was evaporated by gently heating the solution to make a thin film in the vial. To this $1.0 \mathrm{~mL}$ water was added dropwise so that the final concentration of $\mathbf{P 2}$ in water becomes $0.125 \mathrm{mg} / \mathrm{mL}$. The aqueous solution was further sonicated to break any pre-aggregated structure and annealed at $50{ }^{\circ} \mathrm{C}$ in water bath for $3 \mathrm{~h}$ and then cooled to room temperature to obtain a clear solution. The solution was allowed to equilibrate at room temperature for 2 .h before any physical studies were performed. This protocol for sample preparation was followed in each experiment unless specified. For comparing the difference in the self-assembly between annealed and non-annealed samples, the annealing step was avoided in a few experiments. The sample preparation condition was specified in those experiments separately.

UV-Vis studies: Solvent-dependant UV-Vis absorption spectroscopy experiments were performed using a quartz cuvette of $2.0 \mathrm{~mm}$ path length at $25^{\circ} \mathrm{C}$. For variable temperature UVVis studies, the solutions of $\mathbf{P 2}(0.125 \mathrm{mg} / \mathrm{mL})$ in water were heated from $25^{\circ} \mathrm{C}$ to $95^{\circ} \mathrm{C}$ at an interval of $5{ }^{\circ} \mathrm{C}$. Before taking the measurements, each time sample was allowed to stand for $1.0 \mathrm{~min}$ after the desired temperature was reached. For determination of critical aggregation 
concentration (CAC) of $\mathbf{P 2}$ in water, absorbance from different concentration of the $\mathbf{P 2}(0.25$ $0.0005 \mathrm{mg} / \mathrm{mL}$ ) was measured in $5 \mathrm{~mm}$ path length cuvette by stepwise dilution from a higher concentration of $\mathbf{P 2}$ in water.

Photoluminescence studies: Samples were prepared in the same way as discussed earlier for the annealed and non-annealed samples. The emission intensity for $\mathbf{P} 2$ solutions were recorded in a quartz cuvette of $10 \mathrm{~mm}$ path length. The excitation wavelength $\left(\lambda_{\mathrm{ex}}\right)$ was maintained at $360 \mathrm{~nm}$. Excitation and emission band width were kept at $5 \mathrm{~nm}$ each. Relative quantum yields $(\phi)$ were measurements from the solution of $\mathbf{P 2}(0.0625 \mathrm{mg} / \mathrm{mL})$ in its molecularly-dissolved state in $\mathrm{CHCl}_{3}$ and self-assembled state in water with respect to a reference solution of Quinine Sulphate in $0.1 \mathrm{M} \mathrm{H}_{2} \mathrm{SO}_{4}$. The measurements were done following a literature procedure. ${ }^{3}$ using the following equation:

$$
Q_{S}=Q_{R} x \frac{I_{S}}{I_{R}} x \frac{A_{R}}{A_{S}} x \frac{\eta_{S}{ }^{2}}{\eta_{R}{ }^{2}}
$$

where $Q_{S}$ and $Q_{R}$ are the quantum yields of the sample and the reference, respectively. IS and $I_{R}$ stand for the integrated fluorescence intensity area of the sample and the reference, respectively. It is determined from the area under the PL curve of the sample and reference. As and $A_{R}$ are referred to the absorbance of the sample and the reference, respectively. $\eta_{S}=$ refractive index of the sample; $\eta_{R}=$ refractive index of reference. Q.Y. of quinine sulphate was taken as 0.546; Refractive Index of water $=1.33$. This value was used for both $\eta_{S}$ and $\eta_{R}$. The concentration of quinine sulphate was adjusted so that its absorbance was below 0.1 under the experimental condition.

Dynamic light scattering studies: Experiments were carried out with a solution of P2 $(0.125$ $\mathrm{mg} / \mathrm{mL}$ ) in water. The solutions were prepared following the procedure described earlier for annealed and non-annealed samples. Samples were filtered by 0.45 -micron filters before taking the reading. For variable temperature studies, the solution was heated from $20{ }^{\circ} \mathrm{C}$ to $80{ }^{\circ} \mathrm{C}$ at an interval of $10^{\circ} \mathrm{C}$.

Cryo-Transmission Electron Microscopy (Cryo-TEM) studies: $0.5 \mathrm{mg} / \mathrm{mL}$ solution of P2 in water was prepared in the same way as discussed earlier.

High Resolution-Transmission Electron Microscopy (HR-TEM) studies: $0.125 \mathrm{mg} / \mathrm{mL}$ solution of $\mathbf{P 2}$ in water was treated with $35 \%$ THF (v/v). A measured aliquot of the mixture was drop casted on a copper grid and slowly air dried for $24 \mathrm{~h}$ prior to imaging. 
Fluorescence microscopy (FM) studies: A solution of $\mathbf{P 2}(0.125 \mathrm{mg} / \mathrm{mL})$ in water was dropcasted on a glass slide and air dried overnight before FM images were captured.

Förster Resonance Energy Transfer (FRET) Studies: Stock solutions of the donor dye DiO (3,3'-di-octadecyloxacarbocyanine perchlorate) and the acceptor dye DiI (1,1'-dioctadecyl3,3,3', $3^{\prime}$-tetramethylindocarbocyanine perchlorate) were prepared in THF $\left(1 \times 10^{-3} \mathrm{M}\right) .10 \mu \mathrm{L}$ each of $\mathrm{DiO}$ and DiI stock solutions were taken together in a single vial. To this, measured quantity $(0.125 \mathrm{mg})$ of $\mathbf{P 2}$ in $\mathrm{CHCl}_{3}$ was added and the organic solvent was evaporated by heating to make a thin film. This was followed by dropwise addition of $1.0 \mathrm{~mL}$ water so that the final P2 concentration becomes $0.125 \mathrm{mg} / \mathrm{mL}$ and co-encapsulated $\mathrm{DiO}$ and DiI concentration $=1 \times 10^{-5} \mathrm{M}$ each. The resultant aqueous solution of $\mathbf{P 2}$ co-encapsulated with the two dyes was sonicated for 30 mins followed by annealing for $3 \mathrm{~h}$ at $50{ }^{\circ} \mathrm{C}$ in a water bath. Finally, the solution was equilibrated for $2 \mathrm{~h}$ and filtered to remove any non-encapsulated dye. Final dye concentration was maintained at $1.0 \times 10^{-5} \mathrm{M}$ each. For a control experiment, a1:1 mixture of $\mathrm{DiO}$ and $\mathrm{DiI}$ were prepared in water (dye conc. $=1.0 \times 10^{-5} \mathrm{M}$ each) following the same procedure in absence of $\mathbf{P 2}$. To monitor the evolution of the FRET process, the emission spectra were recorded at the donor (DiO) excitation and the acceptor (DiI) emission from these two aqueous solutions (in presence and absence of P2) were compared.

- Dilution Experiment with THF: Similar procedure was followed for the sample preparation as mentioned above for co-encapsulating $\mathrm{DiO}$ and DiI within the nanoparticles of P2. The final dye concentration was maintained at $1.0 \times 10^{-5} \mathrm{M}$ each and $\mathbf{P} 2$ concentration at $0.125 \mathrm{mg} / \mathrm{mL}$. To this solution, measured volume of THF was added stepwise and the emission spectra were recorded at the DiO excitation, to monitor the FRET process.

- Dilution Experiment with water: Similar procedure was followed for the sample preparation as done for dilution experiment with THF. Final P2 concentration $=0.125$ $\mathrm{mg} / \mathrm{mL}$ and co-encapsulated $\mathrm{DiO}$ and DiI concentration $=1 \times 10^{-5} \mathrm{M}$ each. To this solution, measured volume of water was added stepwise and the emission spectra were recorded to monitor the FRET process.

- Kinetic Study: Stock solutions of DiO and DiI were prepared separately in THF $(1 \times$ $\left.10^{-3} \mathrm{M}\right) .10 \mu \mathrm{L}$ each of $\mathrm{DiO}$ and $\mathrm{DiI}$ stock solutions were taken in two separate vials. To these two vials, measured quantity $(0.125 \mathrm{mg})$ of $\mathbf{P 2}$ was added and the organic solvent was evaporated by heating followed by dropwise addition of $1.0 \mathrm{~mL}$ water to make the final polymer concentration $0.125 \mathrm{mg} / \mathrm{mL}$. Both the solutions were sonicated 
for $30 \mathrm{mins}$ and annealed for $3 \mathrm{~h}$ at $50{ }^{\circ} \mathrm{C}$ in water bath. Finally, the solutions were equilibrated for $2 \mathrm{~h}$ and filtered to remove any non-encapsulated dye. Final dye concentration was maintained at $1.0 \times 10^{-5} \mathrm{M}$ in the two individual solutions. $500 \mu \mathrm{L}$ each of individual DiO and Dil loaded P2 $(0.125 \mathrm{mg} / \mathrm{mL})$ solutions were mixed in 1:1 ratio and emission spectra were recorded to monitor the evolution of FRET with time.

Time-Correlated Single Photon Counting (TCSPC) measurements: The intensity average lifetime ( $\tau_{\text {avg }}$ ) of $\mathbf{P 2}$ in the aggregate state and in molecularly dissolved state were determined from their respective solutions in water and $\mathrm{CHCl}_{3}$, respectively. Concentration of $\mathbf{P 2}$ was maintained at $0.0625 \mathrm{mg} / \mathrm{mL}$. The lifetimes were calculated using the following equation. ${ }^{4}$

$$
\tau_{\text {avg }}=\frac{\sum a_{i} \tau_{i}^{2}}{\sum a_{i} \tau_{i}}
$$

where " $\alpha$ " represents the decay contribution corresponding to each lifetime $(\tau)$. $\lambda_{\mathrm{ex}}=375 \mathrm{~nm}$, $\lambda_{\mathrm{em}}=469 \mathrm{~nm}$.

\begin{tabular}{|l|c|c|c|c|c|c|c|l|}
\hline & $\tau_{1}$ & $\tau_{2}$ & $\tau_{3}$ & $\alpha_{1}$ & $\alpha_{2}$ & $\alpha_{3}$ & $X^{2}$ & $\tau_{\text {avg }}(\mathrm{ns})$ \\
\hline $\mathbf{P 2}$ in water & 1.10 & 4.23 & 0.018 & 13.79 & 47.29 & 38.92 & 1.11 & 3.99 \\
\hline $\mathbf{P 2}$ in $\mathrm{CHCl}_{3}$ & 0.574 & 2.572 & 0.04 & 19.03 & 27.65 & 53.32 & 1.17 & 2.24 \\
\hline
\end{tabular}




\section{Additional Figures:}

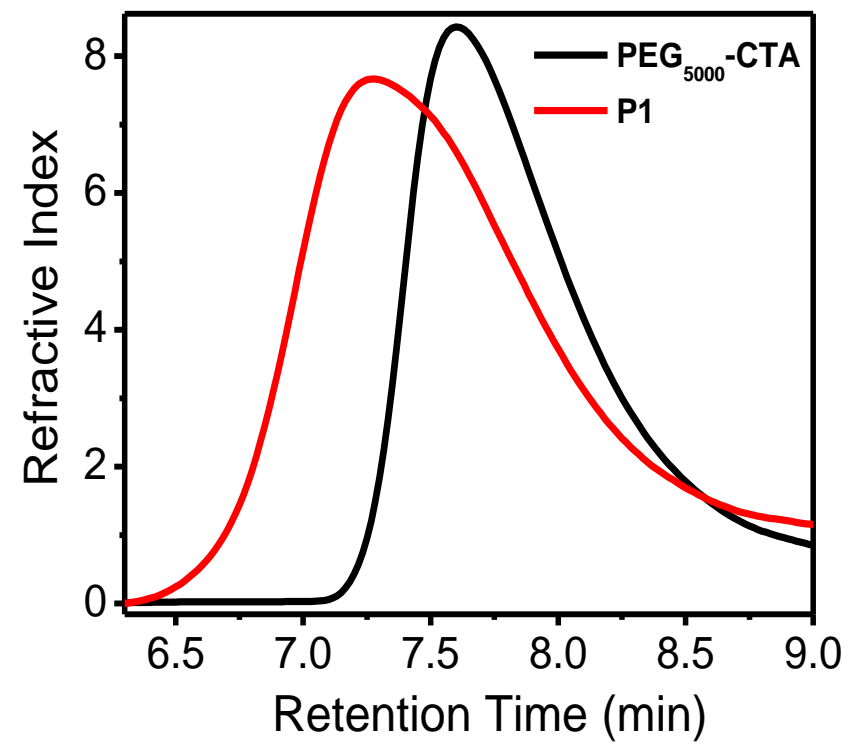

Figure S1. Size exclusion chromatogram in THF.

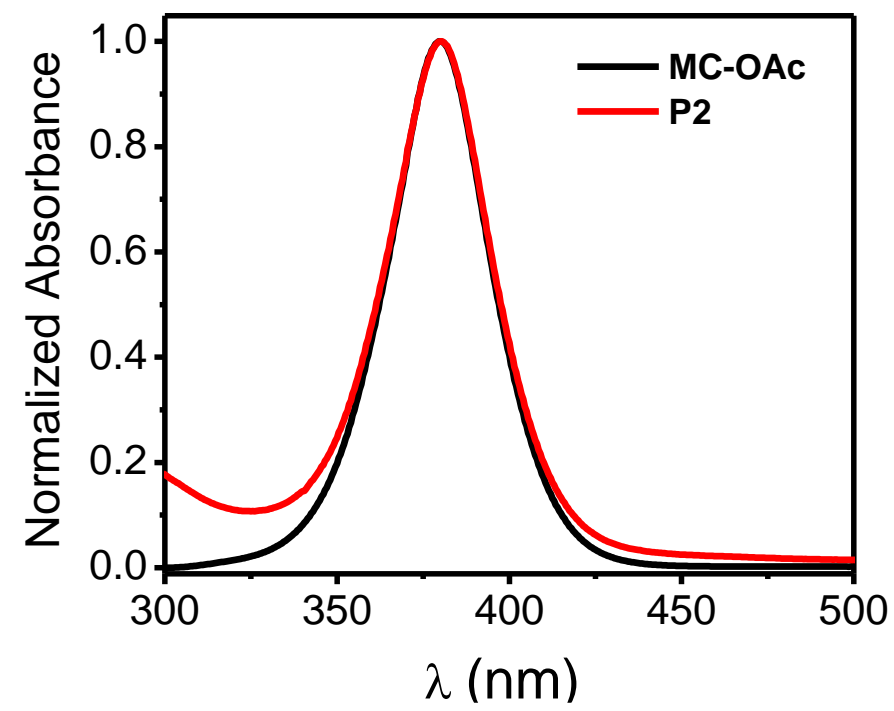

Figure S2. Normalized UV-Vis absorption spectrum of P2 and MC-OAc (acetylated MC$\mathbf{O H})$ in $\mathrm{CDCl}_{3}$. 

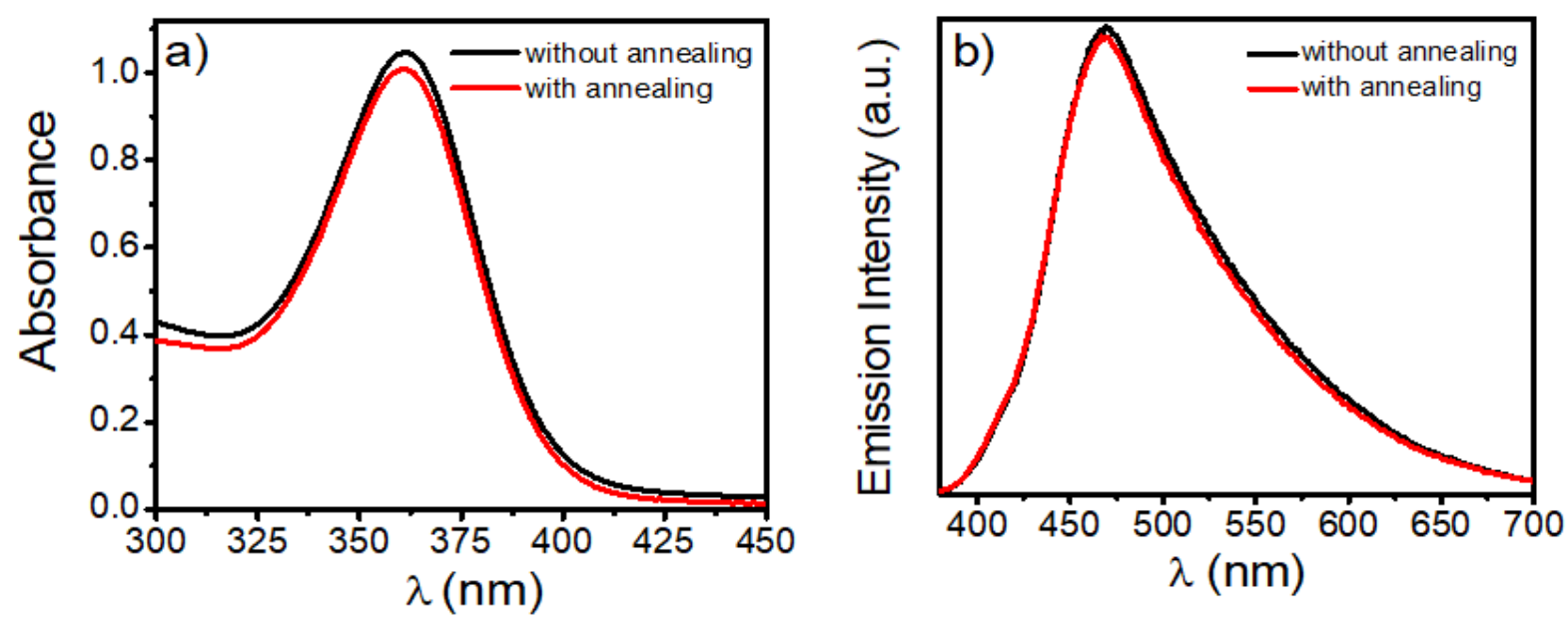

Figure S3. (a) UV-Vis absorption and (b) emission spectra of the aqueous solution of $\mathbf{P 2}$ before and after annealing. Conc. of $\mathbf{P 2}=0.125 \mathrm{mg} / \mathrm{mL} ; \lambda_{\mathrm{ex}}=360 \mathrm{~nm}$; excitation and emission slit $=$ $5 \mathrm{~nm}$ each.

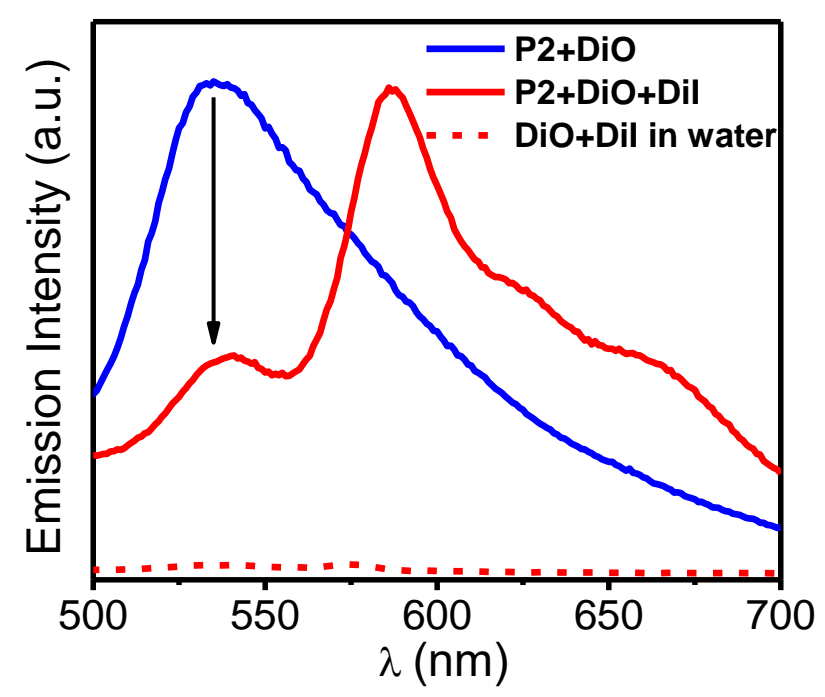

Figure S4. (a) Emission spectra of 1:1 mixture of DiI+DiO in water and co-encapsulated within P2 nanodiscs. DiO emission is significantly reduced when co-encapsulated with DiI, suggesting energy transfer from DiO to DiI. $\lambda_{\mathrm{ex}}=480 \mathrm{~nm}$. Conc. of $\mathrm{DiO}=\mathrm{DiI}=1 \times 10^{-5} \mathrm{M} ; \mathbf{P 2}$ $=0.125 \mathrm{mg} / \mathrm{mL}$; excitation and emission slit $=5 \mathrm{~nm}$ each. 


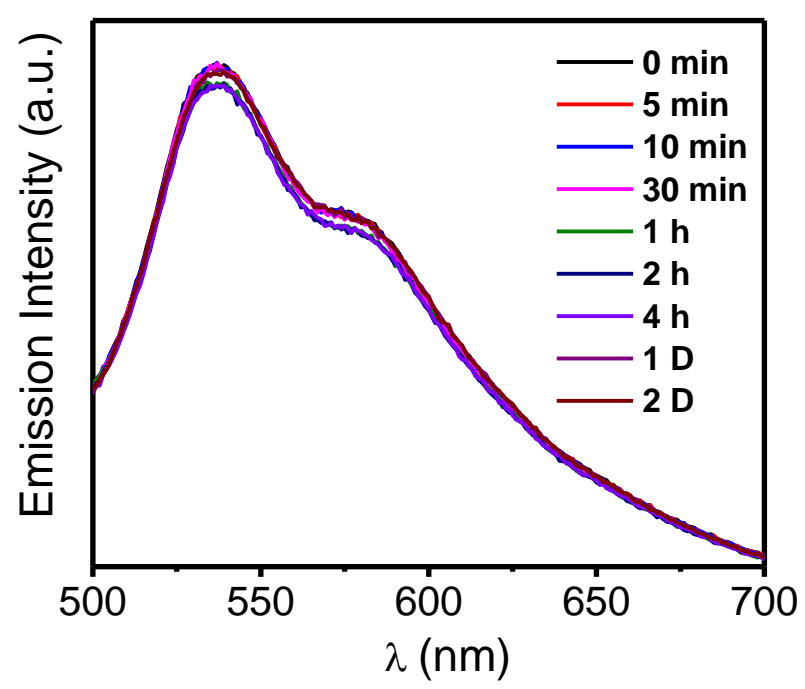

Figure S5. Emission spectra of 1:1 mixture of $\mathbf{P 2}$ nanoparticles in water separately encapsulated with $\mathrm{DiI}$ and DiO. No change in the DiO emission intensity was noted with time. $\lambda_{\mathrm{ex}}=480 \mathrm{~nm}$. Conc. of $\mathrm{DiO}=\mathrm{DiI}=1 \times 10^{-5} \mathrm{M} ; \mathbf{P} 2=0.125 \mathrm{mg} / \mathrm{mL}$; excitation and emission slit $=5 \mathrm{~nm}$ each.
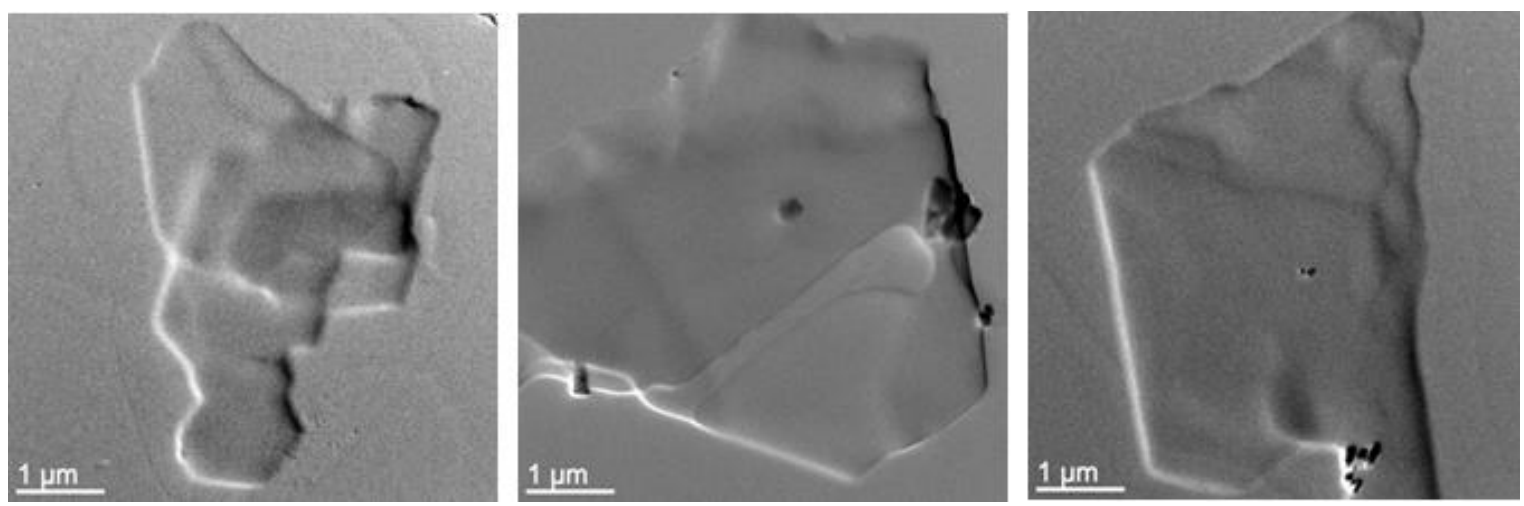

Figure S6. TEM image of $\mathbf{P 2}$ in water in the presence of $35 \%$ THF $(v / v)$. Conc. of $\mathbf{P 2}=0.125$ $\mathrm{mg} / \mathrm{mL}$. 

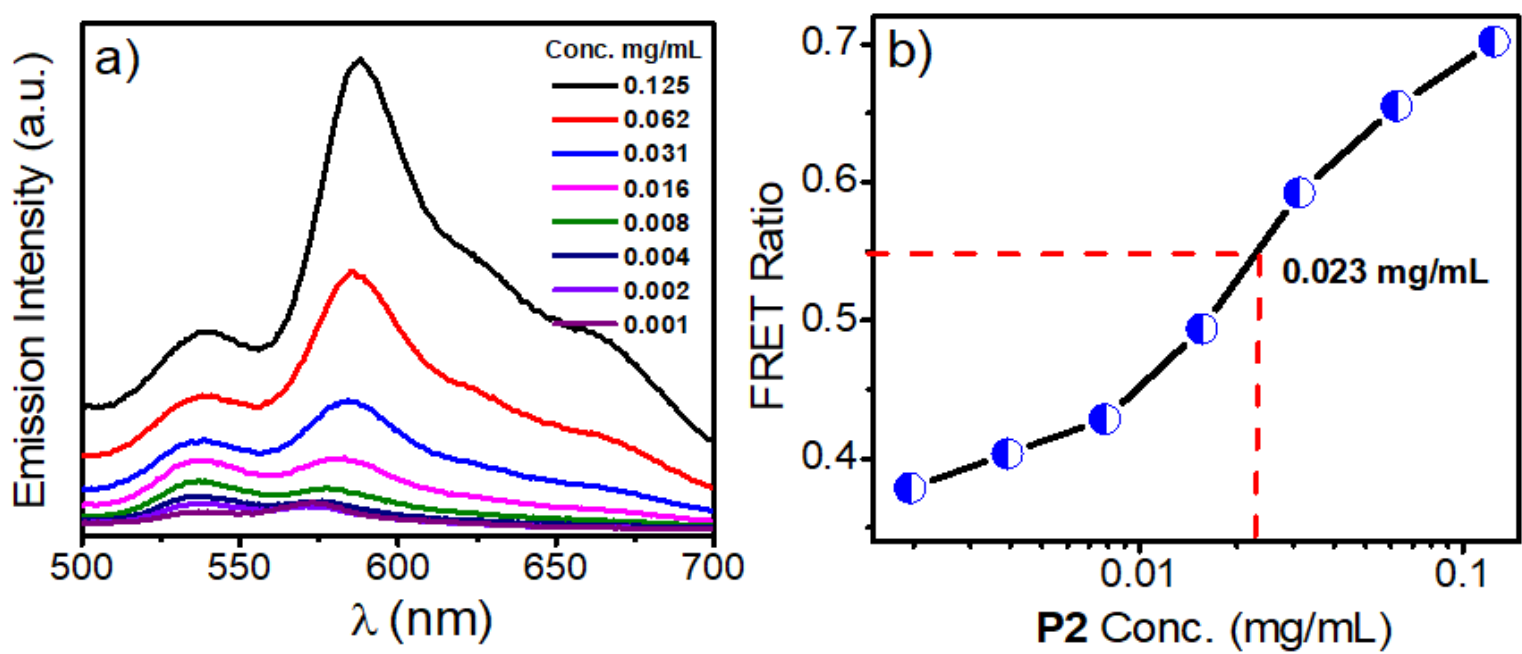

Figure S7. (a) Emission spectra of DiI $+\mathrm{DiO}$ coencapsulated $\mathbf{P 2}$ in water as a function of $\mathbf{P 2}$ concentration. $\lambda_{\mathrm{ex}}=480 \mathrm{~nm}$; excitation and emission slit $=5 \mathrm{~nm}$ each. (b) variation of FRET ratio with concentration.
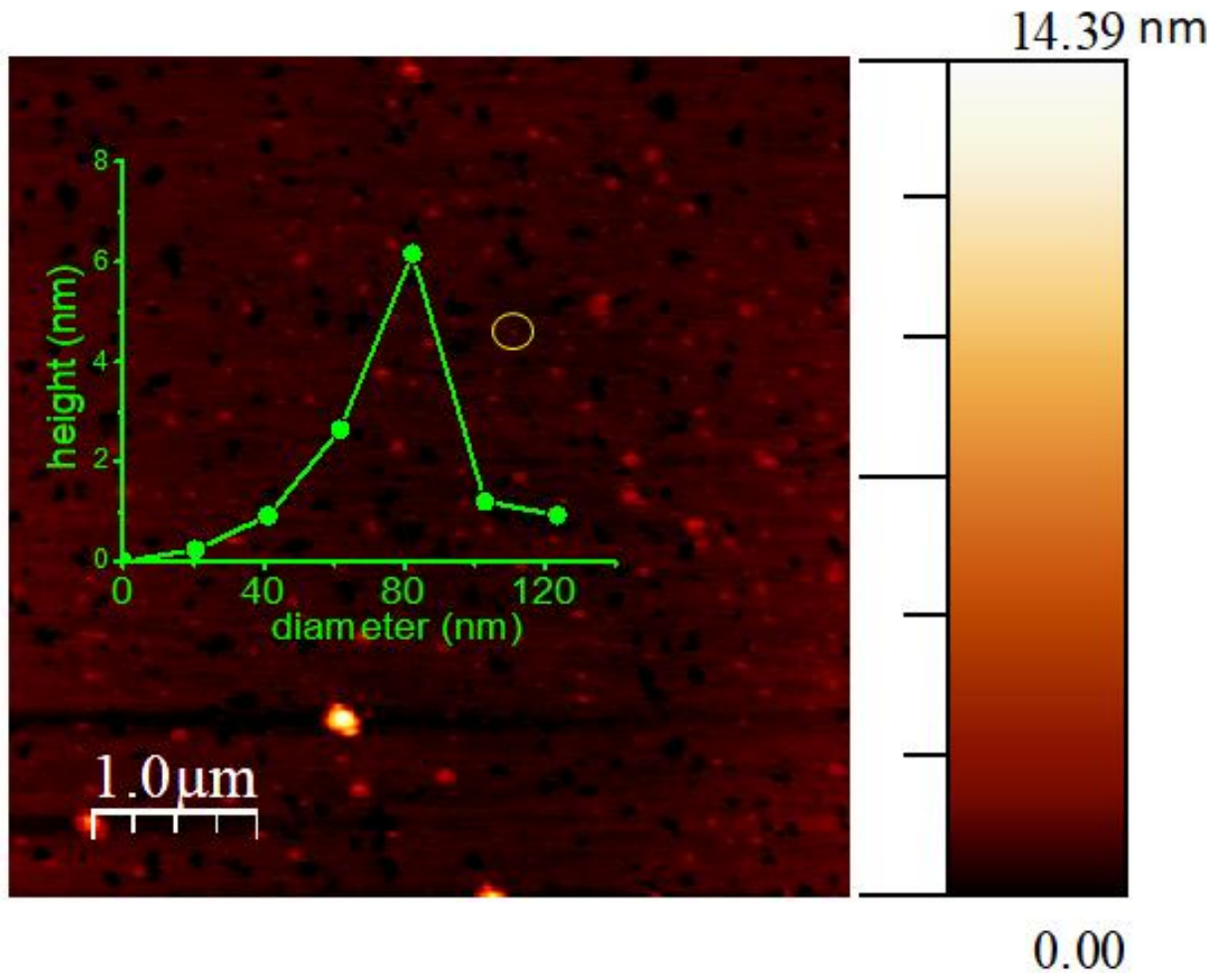

Figure S8. AFM images from a solution of $\mathbf{P 2}$ in water below CAC $(0.005 \mathrm{mg} / \mathrm{mL})$ showing irregularity in particle size and shape. Inset showing the height profile of a smaller aggregate. 


\section{${ }^{1}$ H NMR Spectra:}

${ }^{1} \mathrm{H}$ NMR spectrum of P1; (*) denotes residual solvent peak from $\mathrm{CDCl}_{3}$.

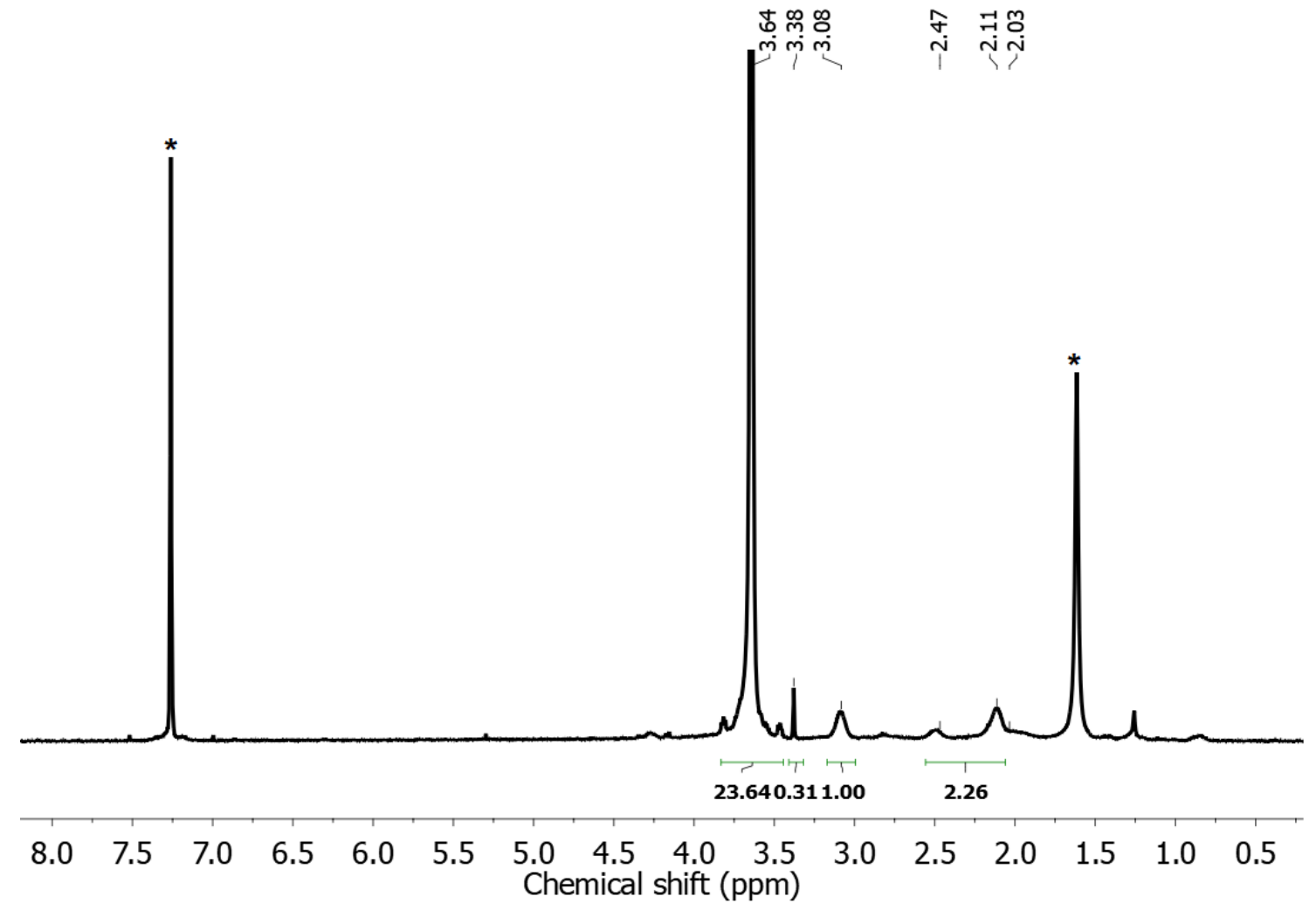

${ }^{1} \mathbf{H}$ NMR spectrum of P2; (*) denotes residual solvent peak from $\mathrm{CDCl}_{3}$.

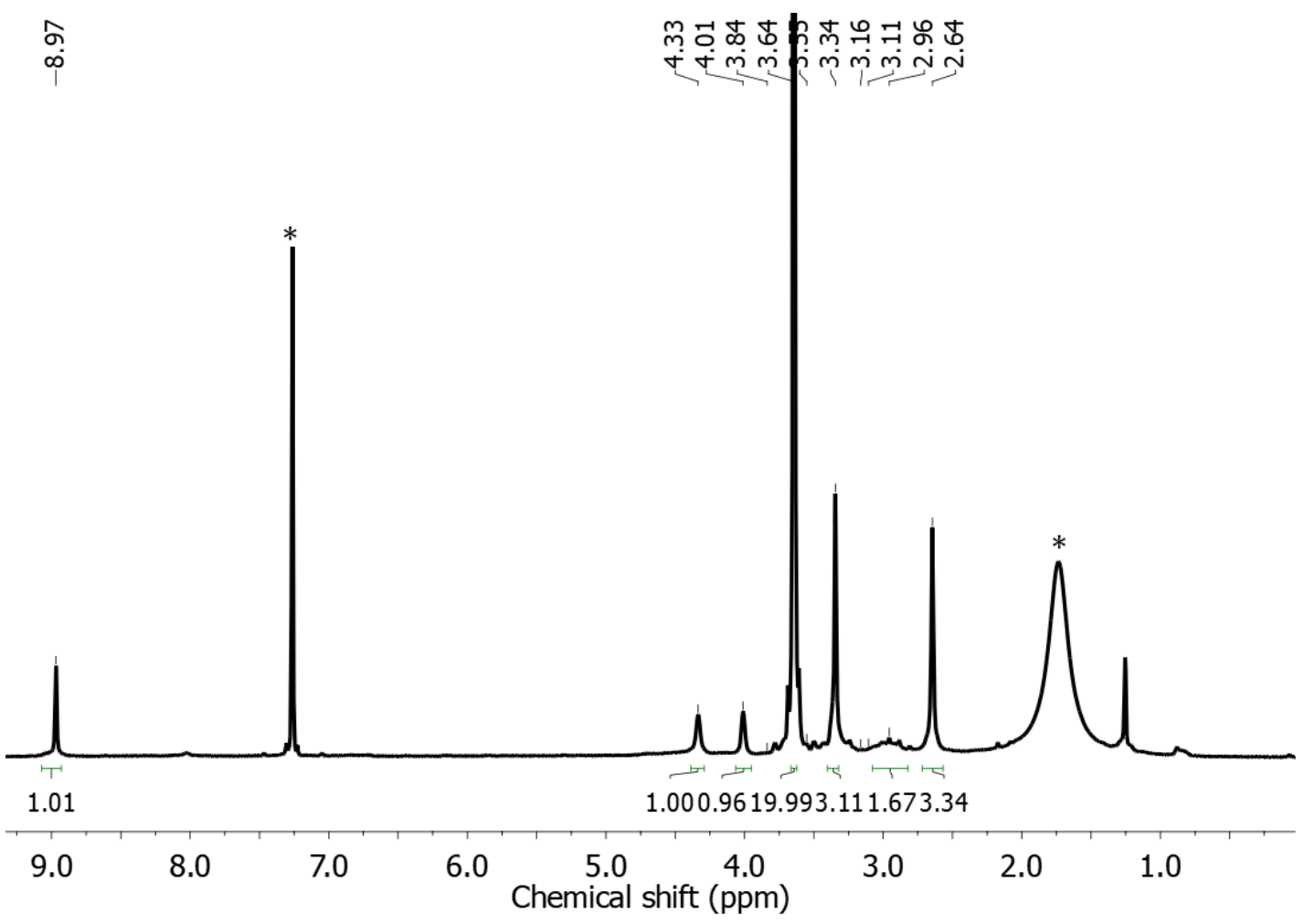




\section{References}

1. Rajak, A.; Karan, C. K.; Theato, P.; Das, A. Supramolecularly cross-linked amphiphilic block copolymer assembly by the dipolar interaction of a merocyanine dye. Polym. Chem. 2020, 11, 695-703.

2. Das, A.; Lin, S.; Theato, P. Supramolecularly Cross-Linked Nanogel by Merocyanine Pendent Copolymer. ACS Macro Lett. 2017, 6, 50-55.

3. a) Sk, M. P.; Chattopadhyay, A. Induction coil heater prepared highly fluorescent carbon dots as invisible ink and explosive sensor. RSC Adv. 2014, 4, 31994-31999.

4. Xie, Y.; Arno, M. C.; Husband, J. T.; Torrent-Sucarrat, M.; O’Reilly, R. K. Manipulating the fluorescence lifetime at the sub-cellular scale via photo-switchable barcoding. Nat.

Commun. 2000, 11, 2460. 